\title{
On the Use of High Magnetic Field in Reactor Grade Tokamaks
}

\author{
Hartmut Zohm ${ }^{1}$ \\ Published online: 9 August 2018 \\ (C) The Author(s) 2018
}

\begin{abstract}
A 0-D model is developed to explore, from the physics point of view, the design options for future reactor grade tokamaks at values of the confining magnetic field exceeding the present technology. It is found that steady state devices with consistent exhaust parameters can indeed be designed at more compact geometry than presently envisaged, but the plasma performance, in particular the stability, is still at the upper end of what has been achieved in present day experiments, i.e. requires an 'advanced tokamak' approach.
\end{abstract}

Keywords Fusion power plant · Tokamak · Magnetic confinement

\section{Introduction}

The performance of magnetically confined fusion plasmas usually increases with increasing magnetic field $B$ for given size, expressed by the torus major radius $R$. Simple scalings [1] show that the fusion power scales as $B^{4} R^{3}$, and the power amplification $\mathrm{Q}$ increases with $1 /\left(B^{-3.7} R^{-2.7}\right.$-const $)$ so that conversely, the major radius $R$ can be decreased if $B$ is increased according to these relations, keeping the plasma performance constant. This has even been interpreted misleadingly as 'fusion performance not depending on size' [2, 3]. Present designs for next step reactor grade experimental devices differ strongly in their assumptions about the technically achievable toroidal field. Relying either on ITER technology $\left(\mathrm{Nb}_{3} \mathrm{Sn}\right)$, the value of $B_{\text {max }}$ at the inner leg of the TF conductor of the order of $12 \mathrm{~T}$ [4]), or new High $\mathrm{T}_{\mathrm{c}}$ (HTSC) superconductors of the REBCO type that could allow up to $24 \mathrm{~T}$ at the inner leg [5], although this value would, using present technology, require exceedingly large support structure to withstand the forces.

This contribution aims at analyzing the principal merits of high field tokamaks (where the term 'high field' means a field above the field possible using state-of the-art ITER

Hartmut Zohm

zohm@ipp.mpg.de

1 Max-Planck-Institut für Plasmaphysik, 8748 Garching, Germany technology) and the new challenges arising, e.g. for exhaust of power through a poloidal divertor which can be more challenging in a compact device. We explicitly leave out a discussion of the technological challenges on the route to using HTSC in fusion, but remind the reader that solving these issues will be a pre-requisite for using high field tokamaks as FPPs and remains a serious R\&D task (at present, there is no convincing demonstration of a HTSC high field solution on the scale needed for a reactor-grade device).

In "The 0-D Model" section, we describe the 0-D model used for the analysis, an extension of the model used in [6] for application in a wider parameter range. In "Exploration of High Field Solutions" section, we discuss suitable figures of merit for quantifying the gain that high field operation may bring and analyze several routes to tokamak FPPs for their prospects on the high field path. In "Discussion and Conclusions" section, a concluding discussion is given.

\section{The 0-D Model}

We use a 0-D model based on the equations presented in [6], improved to explore a larger parameter space. This required to update the calculation of the fusion power for higher temperatures as well as a more detailed radiation model, including separately Bremsstrahlung, impurity radiation and synchrotron radiation (which specifically becomes important when exploring high field solutions). 
In the model, we prescribe the following main plasma parameters (for a definition of the different quantities, see [6]:

- normalized pressure $\beta_{N}$, related to ideal MHD stability,

- $\quad$ safety factor $q_{95}$, related to the plasma current

- normalized confinement time $H=\tau_{E} / \tau_{E, I T E R 98 p}$, assuming that confinement scales similar to the ITER H-mode scaling

- normalized density $f_{G W}=n / n_{G W}$.

Together with the machine geometry parameters, major radius $R,{ }^{1}$ aspect ratio $A$ and toroidal magnetic field $B$, these allow the calculation of fusion power $P_{f u s}$, radiation losses $P_{\text {rad }}$ and auxiliary power needed to sustain the power balance or current drive power $P_{C D}$ needed to drive the difference between total current and bootstrap current, i.e. for fully non-inductive operation.

The formula for the fusion power used previously assumed a quadratic dependence on $\beta_{\mathrm{N}}$, i.e. a scaling with $n^{2} T^{2}$. It is well known that the $T^{2}$ dependence of the reactivity $R_{D T}$ is only valid in the temperature range around $10-20 \mathrm{keV}$. Since FPP designs often exceed this temperature, we use the fit formula derived for $R_{D T}$ in [7], divided by $T^{2}$, so that the equation for the fusion power becomes

$$
\begin{aligned}
P_{f u s}= & c_{1}\left(1-2 f_{H e}-Z_{X e} f_{X e}\right)^{2} \beta_{N}^{2} \frac{R_{D T}}{T^{2}} \frac{B^{4} R^{3}}{q_{95}^{2} A^{4}} \\
& \times\left[\mathrm{MW}, \mathrm{keV}, 10^{20} \mathrm{~m}^{-3}, \mathrm{~T}, \mathrm{~m}\right]
\end{aligned}
$$

The function $R_{D T} / T^{2}$, normalized to its maximum value, is shown in Fig. 1. Chosing $T=1.3 T_{\text {ave }}$, where $T_{\text {ave }}$ is the volume averaged temperature derived via

$T_{\text {ave }}=\frac{1}{c_{3} c_{5}} \frac{2}{2-f_{H e}-\left(Z_{X e}-1\right) f_{X e}} \beta_{N} \frac{B^{2}}{A q_{95} n_{e}}$,

we can reproduce well the fusion power for the three machines studied in [6], i.e. the model is suited well for the kinetic profile shapes used in [6]. On the other hand, we benchmarked the model to ARC [5] and found that we had to increase the coefficient $c_{1}$ by a factor of 1.3 , which could be explained by the relatively broad temperature profiles used in [5] as compared to the cases in Table 1 as well as the different shape of the poloidal cross section.

In Eqs. (1) and (2), we have accounted for the dilution due to Helium ash and the Xenon seed impurities which are assumed to be injected to increase the radiation from inside the separatrix (no other impurities are considered in this work). Both densities are normalized to the electron density, i.e. $f_{H e}=n_{H e} / n_{e}$ and $f_{X e}=n_{X e} / n_{e}$. The quantity $Z_{X e}$ is the average charge state of Xenon in the core plasma (He is fully ionized under these conditions). We note that we do

\footnotetext{
${ }^{1}$ Plasma shape is assumed to be that of ITER so that the volume can be calculated as $\mathrm{V}=\mathrm{V}_{\text {ITER }}(\mathrm{R} / 6.2 \mathrm{~m})^{3} /(\mathrm{A} / 3.1)^{2}$.
}

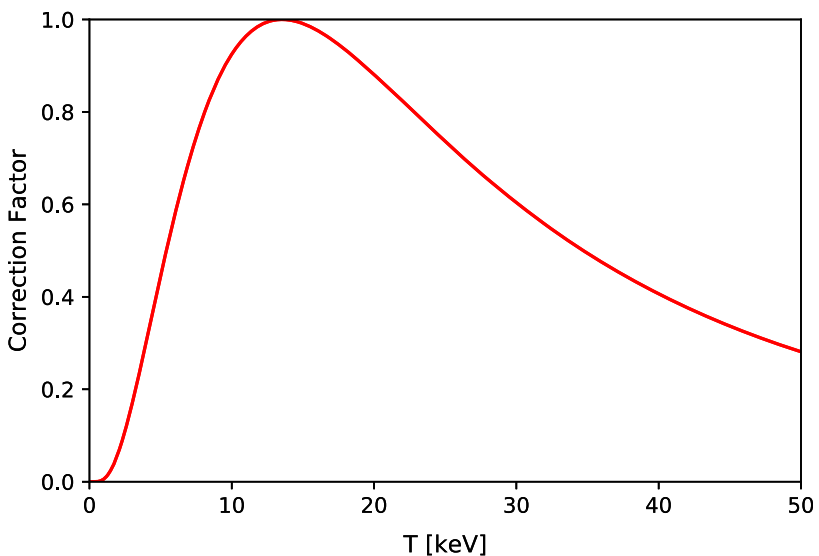

Fig. 1 The function $R_{D T} / T^{2}$ used to correct the reactivity from the simple $T^{2}$ scaling

not correct the heating power for the radiation losses, motivated by the finding that for stiff temperature profiles, confinement is hardly affected by radiation losses as long as $P_{\text {rad }}(r)$ does not overlap with $P_{f u s}(r)$ [8], which is the case for Xenon for typical profiles in reactor grade devices that tend to be peaked off-axis [8].

The radiation model used in [6] was very simplistic, assuming a direct scaling of the radiated power with $Z_{\text {eff. }}$ In the new version, we explicitly separate the Bremsstrahlung

$P_{B r}=5.35 \times 10^{-3} G Z_{e f f} n_{e}^{2} \sqrt{T_{a v e}} V$

where $\mathrm{G}$ is the Gaunt factor (set to 1.1 in this work), $V$ is the plasma volume and the average charge is calculated as

$Z_{\text {eff }}=1+2 f_{H e}+\left(Z_{X e}^{2}-Z_{X e}\right) f_{X e}$.

As mentioned above, $\mathrm{Xe}$ is used as seed impurity used for controlling the core radiation and assumed to dominate the impurity radiation so that no other impurity is considered. The line radiation due to $\mathrm{Xe}$ is inferred using the radiative potential $L_{Z}(T)$ :

$P_{\text {line }}=f_{X e} n_{e}^{2} L_{X e}(T) V$.

For the cases treated in [6], which employed Xe as seed impurity, it was found that the main radiation comes from the zone where $10 \mathrm{keV}<T<15 \mathrm{keV}$, for which we can use $L_{X e}=370 \mathrm{MW} \mathrm{m}^{3}$ and $Z_{X e}=50$. Finally, the synchrotron radiation is evaluated as

$P_{\text {syn }}=1.32 \times 10^{-7}\left(B T_{\text {ave }}\right)^{2.5} \sqrt{\frac{A n_{e}}{R}}\left(1+\frac{18}{A \sqrt{T_{\text {ave }}}}\right) V$

according to [9], using a wall reflectivity of 0.8 . The total radiation $P_{\text {tot }}$ is calculated by summing up the three contributions (3), (5) and (6). We determine $f_{X e}$ by the criterion that the power across the separatrix exceeds the $\mathrm{L}-\mathrm{H}$ threshold power by a factor $f_{L H}$ 
Table 1 Input parameters used in the study of the 4 plasma scenarios

\begin{tabular}{lllll}
\hline & ITER Q = 10 & AUG hybrid & Stepladder & DIII-D/EAST \\
\hline $\mathrm{q}_{95}$ & 3.1 & 5.4 & 4.5 & 6.5 \\
$\mathrm{H}$ & 1.0 & 1.1 & 1.2 & 1.5 \\
$\beta_{\mathrm{N}}$ & 1.8 & 2.8 & 3.5 & 3.0 \\
$\mathrm{f}_{\mathrm{GW}}$ & 0.85 & 0.7 & 1.2 & 1.0 \\
\hline
\end{tabular}

$P_{\text {rad }}\left(f_{X e}\right) \stackrel{!}{=} P_{\text {rad }, \text { required }}=\frac{P_{f u s}}{5}+P_{C D}-f_{L H} P_{L H}$,

assuming that $P_{C D}>P_{A U X}$, i.e. always sufficient to guarantee burn. We note that by applying this procedure, the impurity seeding directly feeds back into $P_{C D}$ via the $\left(5+Z_{\text {eff }}\right)$ dependence of

$P_{C D}=\frac{c_{8} c_{3} c_{5} c_{7}^{3}}{\pi^{2}} \frac{B}{q_{95}^{2}} \frac{f_{G W}^{2}}{\beta_{N} A}\left(5+Z_{e f f}\right)\left(1-C_{9} \sqrt{A} q_{95} \frac{c_{4}}{c_{3}} \beta_{N}\right)$

which means $f_{X e}$ has to be determined iteratively. As outlined in [6], Eq. (8) assumes a generic RF current drive efficiency of the form $P_{C D} \sim n R / T$ and should hence be a good approximation for ECCD, but less well suited for NBCD.

\section{Exploration of High Field Solutions}

In this section, we apply the model described in the previous section to study the possible parameter space of future reactor-grade tokamaks allowing high toroidal field and neglecting, for the moment, the present technological limitations to the increase of the field. The study will analyse different routes from present day experiments to reactor grade plasmas, namely the ITER $Q=10$ scenario at low q $\mathrm{q}_{95}$ [10], a 'hybrid'-type steady state scenario demonstrated on ASDEX Upgrade [11] and DIII-D [12] at intermediate $\mathrm{q}_{95}$ as well as a lower $\mathrm{q}_{95}$ version proposed for the 'stepladder' in [6] and a high $\mathrm{q}_{95}$ variant demonstrated on DIII-D and EAST [13] for use on CFETR [14]. In choosing these cases, the study is limited to conventional aspect ratio of $\mathrm{A}=3.1$ (the ITER value is used throughout). A study of compact solutions at low aspect ratio is subject to further work.

Table 1 shows the parameters $q_{95}, H, \beta_{N}$ and $f_{G W}$ for these 4 cases, noting that $f_{H e}=0.05, f_{L H}=1.1$ and $A=3.1$ are kept constant in the study. The values for ITER $\mathrm{Q}=10$, AUG Hybrid and Stepladder have been taken directly from refs $[6,10,11]$, respectively. For the DIII-D/EAST scenario, we took the values for the discharge discussed in [13], and chose $q_{95}$ such that the value of the plasma current is matched. Due to the slightly differing aspect ratio and shape, this leads to a lower value than quoted in [13].

For these cases, we will discuss the following parameters in the $R-B$ plane:

- Fusion power $P_{f u s}$ : even though high field may allow smaller unit sizes, we still anticipate that an FPP will generate several $\mathrm{GW}$ of fusion power in order to arrive at reasonable recirculating power fraction due to the relatively large auxiliary power needed for a tokamak. Hence, we explore $P_{f u s}$ up to $3.5 \mathrm{GW}$, aiming at around $1 \mathrm{GW}$ of electrical power at conventional efficiency.

- Power amplification w.r.t. power balance, $Q_{P B}=P_{f u s}$ $P_{A U X}$ : this shows how close the plasma is to ignition, and how effective it can be in generating electrical power. Assuming that $P_{A U X}$ is the dominant electrical power needed to sustain the plant, the recirculating power is

$f_{\text {rec }}=\frac{P_{e l, A U X}}{P_{\text {el,tot }}}=\frac{1}{1.18 Q_{P B} \eta_{A U X} \eta_{T D}}$

where we have accounted for the thermal power generated in the blanket by nuclear reactions by the factor 1.18 and introduced the efficiencies $\eta_{A U X}$ (wall plug efficiency of the auxiliary heating system) and $\eta_{T D}$ (thermodynamic efficiency to generate electricity from heat). For example, for $\eta_{A U X}=0.4$ and $\eta_{T D}=0.35$, we obtain $f_{\text {rec }}=6 / Q_{P B}$ and a reasonable value of $f_{\text {rec }}$ $<10 \%$ will require $Q_{P B}>60$. We note here that in our approach, for an ignited plasma, there is no attempt to fulfill exactly the power balance, i.e. these cases would strictly not be stationary, but for the scoping studies shown here this is not considered to be too important.

- Power amplification w.r.t. current drive power $Q_{C D}=$ $P_{f u s} / P_{C D}$ : while $Q_{P B}$ is calculated from the power balance, the requirement of steady state will often lead to values of $P_{C D}$ [see Eq. (8)] that exceed $P_{A U X}$ and in this case $Q_{C D}$ will determine the recirculating power fraction, calculated by using $Q_{C D}$ and $\eta_{C D}$ in Eq. (9), where $\eta_{C D}$ is the wall plug efficiency of the CD system (the current drive efficiency in the plasma is already taken into account by calculating $P_{C D}$ according to (8)). Hence, we also map out this quantity in the $R-B$ space, noting that for a pulsed tokamak FPP, this constraint will not exist. In principle, $P_{C D}$ and $P_{A U X}$ should have the same value for a stationary solution, but usually, $P_{C D}>P_{A U X}$ is found. In these cases, the fusion performance might be higher than estimated by our model, since there is excess heating, but as for the ignited cases discussed above, this is not considered to be too important for the scoping studies shown here.

- Contribution of synchrotron radiation: a particularity of high field tokamaks is the possibility of synchrotron 
radiation dominating the power balance. We define as a rough indicator of this the quantity $f_{\text {sync }}=P_{\text {synd }}$ $P_{\text {rad,required }}$, where $P_{\text {rad,required }}$ is defined by Eq. (7). If $f_{\text {sync }}>1$, the radiation power exceeds the required power even at $f_{X e}=0$, i.e. the synchrotron losses are intolerable. Operation above this line will not be possible.

- Similarity in exhaust: in principle, an exhaust solution should be modelled using more sophisticated codes than the one discussed here to find the seed impurity concentration needed in the SOL and divertor to provide a detached solution. This concentration would then have to be fed back to the core plasma, assuming a certain compression ratio. Such a model has been recently developed and applied in [15]. However, this is beyond the scope of the present study. Hence, we rather adopt a similarity criterion put forward in [16], that states that the impurity concentration in the SOL and divertor needed to obtained a detached divertor solution is expected to scale like

$f_{z} \propto \frac{B^{0.88} R^{1.33} q^{0.32}}{f_{G W}^{1.18}}$

at constant $f_{L H}, A$ and shape. Note that fixing $f_{L H}$ means that $f_{X e}$ varies with fusion power, feeding back into the power balance via Eq. (1). This scaling has also been found in [15]. In the following, we use it to connect the existing model points from Table 1 to other points in parameter space, arguing that the exhaust problem will be similar along this line and the solution developed for the points in Table 1 will apply for all points on the line. For scenarios where no exhaust scenario has been studied, we plot an 'ITER Q $=10$ exhaust similarity line'

$B=B_{\text {ITER }}\left(\frac{f_{G W}}{f_{G W ; I T E R}}\right)^{1.34}\left(\frac{q_{95, I T E R}}{q_{95}}\right)^{0.37}\left(\frac{R_{\text {ITER }}}{R}\right)^{1.51}$

These parameters will now be analysed for the 4 cases from Table 1.

We start by discussing the ITER $Q=10$ scenario, shown in Fig. 2. It can be seen that due to the low $q_{95}$, ignition (i.e. large $Q_{P B}$ ) is relatively easy to achieve, e.g. by lowering $R$ to $5 \mathrm{~m}$ and increasing $B$ to $7.4 \mathrm{~T}$. On the other hand, the low $\mathrm{q}_{95}$ leads to a low bootstrap fraction and steady state needs a lot of current drive power. Hence, $\mathrm{Q}_{\mathrm{CD}}$ has low values, hardly exceeding $Q_{C D}=5$, across the whole range under consideration $\left(P_{\text {fus }}<3.5 \mathrm{GW}\right)$. As a result of the substantial $P_{C D}$, the Xe concentration is quite high up to the line where synchrotron radiation takes over, which in this case is at very large values of $B$ and $R$ and has been indicated in the plot by $f_{\text {sync }}=0.8\left(f_{\text {sync }}=1.0\right.$ actually lies outside the plotted window). We conclude that for this scenario, it is not possible to obtain an attractive steady state reactor, even if $B$ is allowed to be increased substantially.

The conclusion for the ITER Q $=10$ point is of course in line with many previous studies and steady state solutions are usually explored at higher $q_{95}$, which will increase the bootstrap fraction, but at the same time, reduce the fusion power at given $\beta_{N}$ and also make ignition harder due to the reduced current.

This route has been explored in the ASDEX Upgrade hybrid scenario [11], and hence, as an example of a direct extrapolation of presently achieved parameters, we plot the $R-B$ space for this set in Fig. 3. It can be seen that the objective of increasing $Q_{C D}$ at reasonable $P_{\text {fus }}$ is met (note the different scale for $Q_{C D}$ w.r.t. Figure 2), i.e. compact high field devices in this scenario can have $Q_{C D}$ around 30 at roughly $2 \mathrm{GW}$ of fusion power. This is mainly due to the combination of a relatively low Greenwald fraction with high $q_{95}$, which leads to low absolute density and very high temperature. In fact, it can be seen that now the synchrotron limit becomes substantial, restricting operational space to $P_{\text {fus }}$ below $2.5 \mathrm{GW}$. We also note that no study of a consistent exhaust scenario exists for this approach and, due to the low absolute density, it may be hard to find detached solutions. In fact, assuming exhaust similarity to ITER Q = 10 using Eq. (11) shows that this is not fulfilled anywhere in the $R-B$ space shown in Fig. 3 .

These limitations have led to the study of the so-called stepladder scenario in [6], which employs higher $f_{G W}$ and lower $q_{95}$, in an attempt to keep the absolute density high and still provide a reasonable bootstrap fraction. For this scenario, it has been argued that the exhaust problem is similar to that of ITER $Q=10$ and hence, the solution developed for this scenario should also apply to the stepladder. In Fig. 4, we show the $R-B$ space for the stepladder scenario and insert 3 blue lines which indicate the steps on the ladder, extrapolated with the exhaust similarity criterion from [16]. The red dots refer to ITER, DEMO and the FPP on the stepladder (ITER is not visible since it has $B=4.5 \mathrm{~T}$ in this scenario).

It can be seen that the approach is indeed successful in avoiding the synchrotron limit and providing reasonable $Q_{C D}$. If one wants to reduce the size of the FPP point along the exhaust similarity line, one quickly enters the region of too high $P_{\text {fus }}$, meaning that this process should start from a smaller machine, e.g. the DEMO point. One notes, however, that this leads into the region of relatively high $f_{X e}$, which has the effect that $Q_{C D}$ does not rise too strongly along this line.

Obviously, a problem in our approach is that the exhaust similarity line does not deviate too strongly from the lines of constant $Q_{C D}$, making it hard to profit from the smaller 
Fig. 2 Contours of $P_{f u s}, Q_{P B}$, $Q_{C D}$ and $f_{X e}$ in the $R, B$ plane for the ITER $Q=10$ scenario. The red dot represents the ITER $Q=10$ operational point, the blue line indicates the exhaust similarity scaling. The green line is the synchrotron limit, in this case taken to be $f_{\text {sync }}=0.8$. Note that the scale for $Q_{C D}$ is a factor of 10 lower than for the following plots, and the scale for $f_{X e}$ is higher by a factor of 3.75 (Color figure online)
Fig. 3 Contours of $P_{f u s}, Q_{P B}$, $Q_{C D}$ and $f_{X e}$ in the $R, B$ plane for the ASDEX Upgrade hybrid scenario. The green line is the synchrotron limit, $f_{\text {sync }}=1$ (Color figure online)
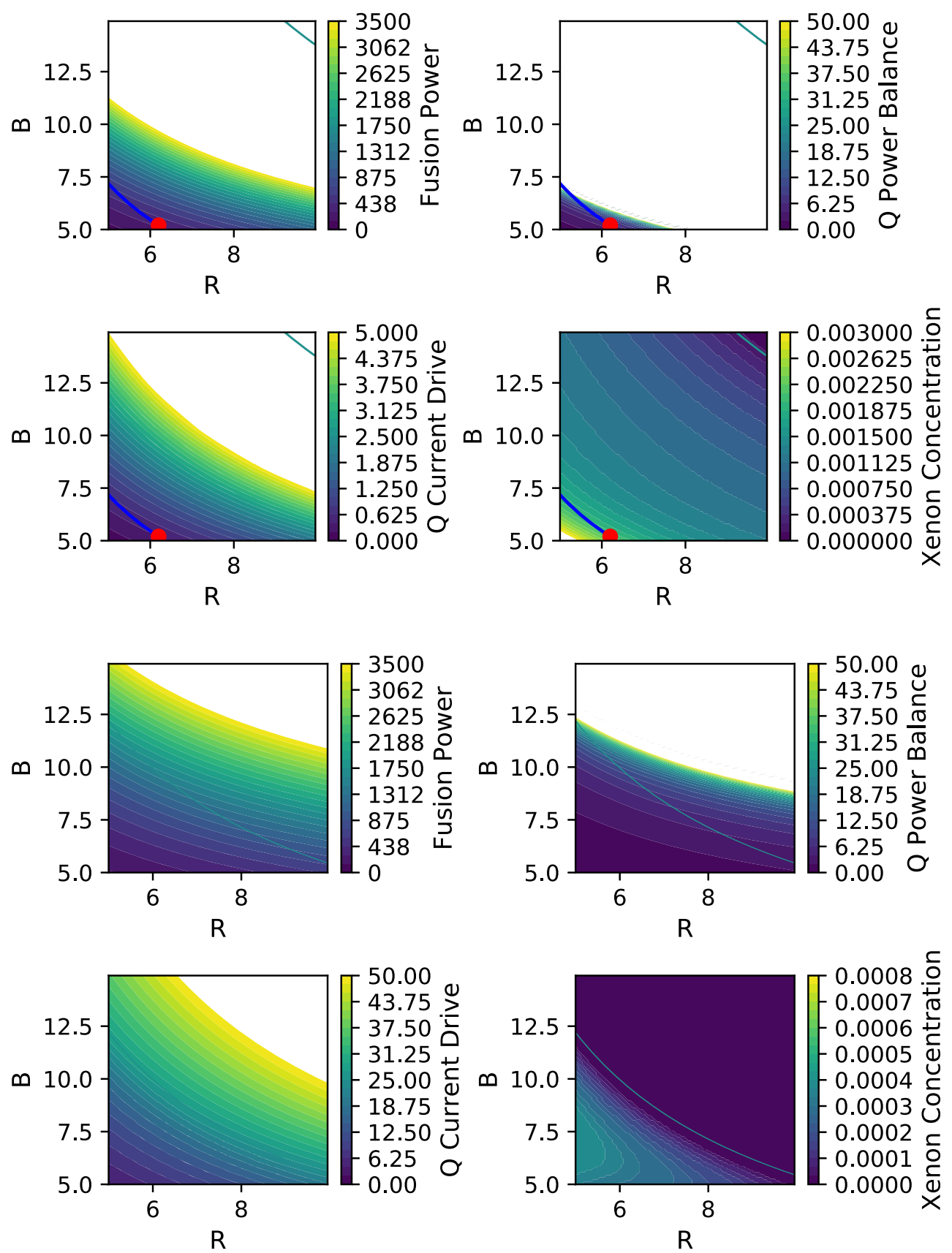

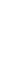


Fig. 4 Contours of $P_{f u s}, Q_{P B}$, $Q_{C D}$ and $f_{X e}$ in the $R, B$ plane for the stepladder scenario. The red dots represent the DEMO and FPP operational points, the blue lines indicates the exhaust similarity scaling for the respective devices. The green line is the synchrotron limit $f_{\text {sync }}$ $=1.0$ (Color figure online)
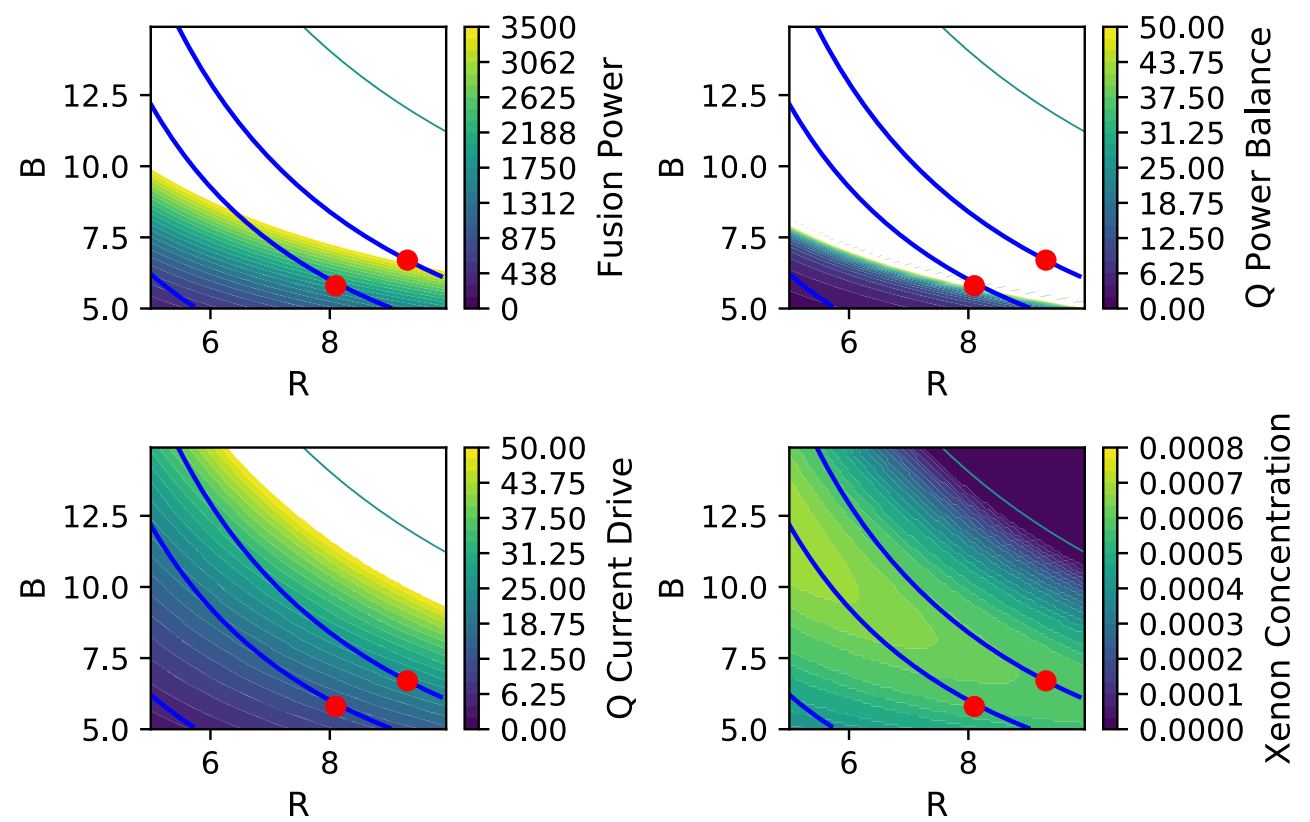

Fig. 5 Contours of $P_{f u s}, Q_{P B}$, $Q_{C D}$ and $f_{X e}$ in the $R, B$ plane for the DIII-D/EAST scenario. The red point represents an examples at $(6 \mathrm{~m}, 10 \mathrm{~T})$ discussed in the text, the blue line indicates the exhaust similarity scaling to the ITER $Q=10$ scenario. The green line is the synchrotron limit $f_{\text {sync }}=1.0$ (Color figure online)
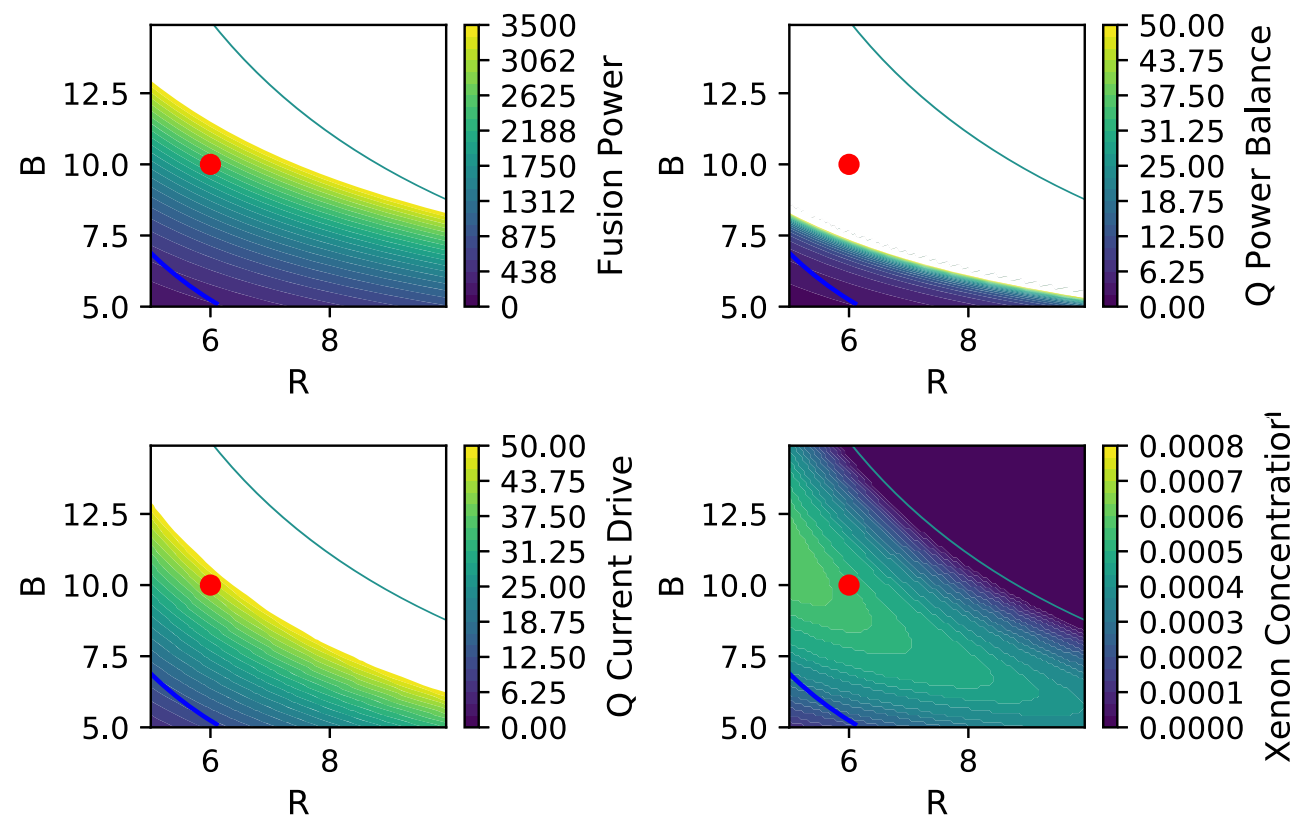

From the plots in Fig. 5, one can see that this indeed represents a step in a direction where $Q_{C D}$ becomes higher, synchrotron radiation is not a problem, and fusion power stays below the $3.5 \mathrm{GW}$ limit. We have highlighted a point at $R=6 \mathrm{~m}, B=10 \mathrm{~T}$, which sits at $Q_{C D}=43$ and $P_{\text {fus }}$ $=2.35 \mathrm{GW}$ and might be an attractive steady state scenario. We note from the plot of $Q_{P B}$ that from the view of power balance, the point already sits deeply in the ignited regime, meaning that the assumption $H=1.5$ could even be relaxed $\left(Q_{C D}=Q_{P B}\right.$ for $H=1.17$ in this case). We also note that no studies of a consistent exhaust scenario exist for this point. The ITER $\mathrm{Q}=10$ exhaust scenario line according to (11) is indicated in blue in the diagram, showing that finding a consistent exhaust scenario for this approach may also be challenging.

\section{Discussion and Conclusions}

In the previous sections, we have analysed how different optimization strategies developed for reactor-grade tokamaks with conventional magnetic field values would 
Fig. 6 Contours of $P_{f u s}, Q_{P B}$, $Q_{C D}$ and $f_{X e}$ in the $R, B$ plane for an scenario optimized for compact steady state tokamak operation at low recirculating power. The red point represents an example at $(5 \mathrm{~m}, 9.3 \mathrm{~T})$ discussed in the text, the blue line indicates the exhaust similarity scaling to the ITER $Q=10$ scenario. The green line is the synchrotron limit $f_{\text {sync }}=1.0$ (Color figure online)
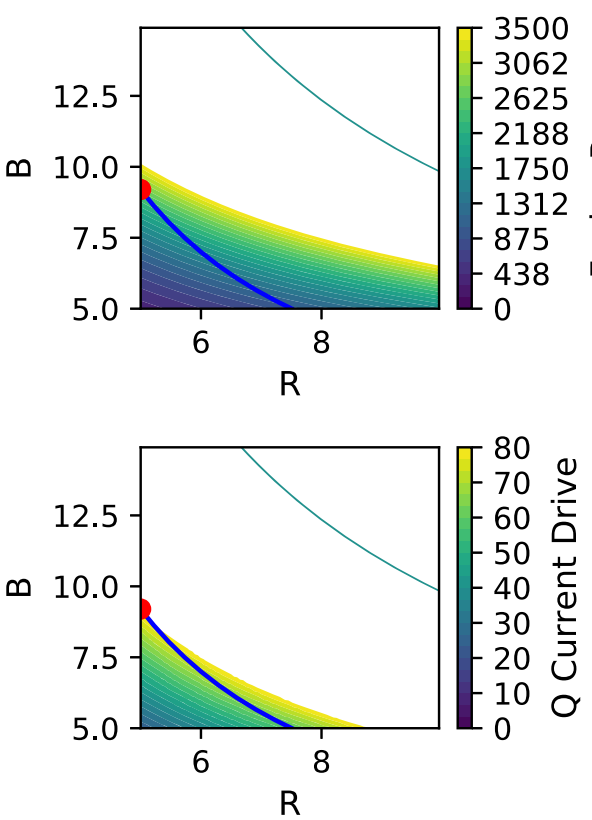
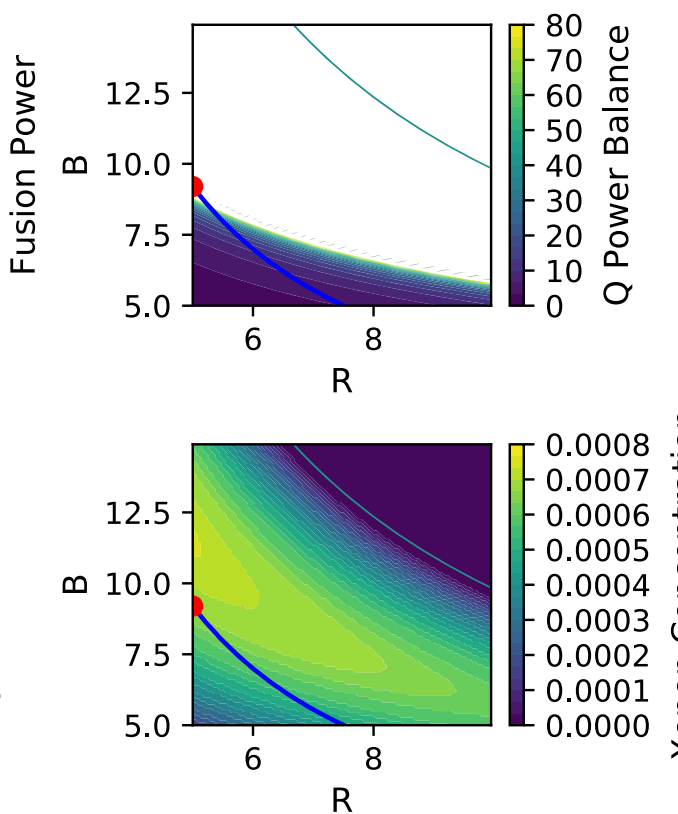

extrapolate to higher B. It is clear that none of these really leads directly to an optimized high field device, since the different approaches encounter various problems:

- a low $\mathrm{q}_{95}$, low $\beta_{\mathrm{N}}$ approach, which is applied to maximize fusion power in pulsed ITER discharges, does not extrapolate to steady state at higher B since the gain in QCD is relatively small when moving on the exhaust similarity line

- in the usual advanced tokamak approach of increasing $q_{95}$ and $\beta_{\mathrm{N}}$ for higher bootstrap fraction and, at the same time, $\mathrm{H}$ to compensate for the lower confinement and higher $\mathrm{q}_{95}$, the Greenwald fraction also has to be increased because otherwise, the absolute density gets so low that no exhaust scenario compatible with present day approaches exists. In addition, high $\mathrm{q}_{95}$ at low $\mathrm{f}_{\mathrm{GW}}$ also leads very high temperatures at which, together with the high field, synchrotron radiation becomes important. On the other hand, high $\mathrm{f}_{\mathrm{GW}}$ will also help solving the exhaust problem.

Hence, the use of high field in an advanced tokamak approach will require high $\mathrm{f}_{\mathrm{GW}}$, an ingredient which is presently not integrated with this approach.

To study a possible target for such a scenario, we show the $R-B$ space for an advanced tokamak $\left(\beta_{N}=4.0, H\right.$ $\left.=1.3, f_{G W}=1.2, q_{95}=5.7\right)$. It can be seen that this choice of parameters leads to an attractive operational point at $R=5 \mathrm{~m}, B=9.3 \mathrm{~T}$, producing $2.7 \mathrm{GW}$ of fusion power at $Q_{C D}=80$, i.e. with the prospect to reach low recirculating power fraction below $10 \%$. Due to the high $f_{G W}$, the average electron density is $1.6 \times 10^{20} \mathrm{~m}^{-3}$ and the average temperature is $20 \mathrm{keV}$, leading to a large margin against synchrotron radiation and compatibility with the ITER exhaust requirements. This point, by no means optimized, can be taken as a start for optimization studies to find how to best exploit the high field.

We note that the plasma parameters assumed in Fig. 7 are still quite challenging, and the question arises if higher $B$ can actually be used to design devices that are attractive with more conservative assumptions about the plasma performance. Figure 7 illustrates the difficulty in finding such a parameter set. In the left part, the plasma parameters from the example in Fig. 6 have been used. The ITER exhaust similarity line is shown in red, and any solution that lies below this line will be acceptable form the exhaust point of view. The blue line shows the line on which $Q_{C D}$ $=80$, and any solution that lies beyond this line is acceptable w.r.t. recirculating power. This defines a region between the two curves in which acceptable solutions can lie. However, we also have to make sure that $Q_{P B}$ is of the order of $Q_{C D}$ to be consistent in the power balance. Hence, we have overplotted the contours of the $\mathrm{H}$-factor needed to obtain $Q_{P B}=80$. Consistent the assumptions used in Fig. 6, the region of interest is accessible with $H$ around 1.2 (the values from Fig. 6 are not precisely matched since we have set $f_{X e}=0$ to be able to invert the equations for the plot).

The right side of Fig. 7 shows a similar approach for more conservative parameters, $\beta_{N}=3.0$ and $f_{G W}=1.0$. Due to the lower $\beta_{N}$, it is difficult to open up a region of interest since for $\mathrm{q}_{95}<7$, there is no overlap at all between the red and the blue line. The case shown in Fig. 7, employing $q_{95}=8.3$, is close to $100 \%$ bootstrap fraction and hence opens up an acceptable region. However, it can be seen that in this region, due to the high $q_{95}$, the required 
Fig. 7 Left: contours of the $\mathrm{H}$-factor needed for $Q_{P B}=80$ for the parameter set from Fig. $6\left(\beta_{N}=4.0, f_{G W}=1.2\right.$, $\left.q_{95}=5.7\right)$. The red line shows the ITER exhaust similarity line, the blue line the line on which $Q_{C D}=80$. Right: the same for a more conservative set $\left(\beta_{N}=3.0, f_{G W}=1.0\right.$, $\left.q_{95}=8.3\right)($ Color figure online)

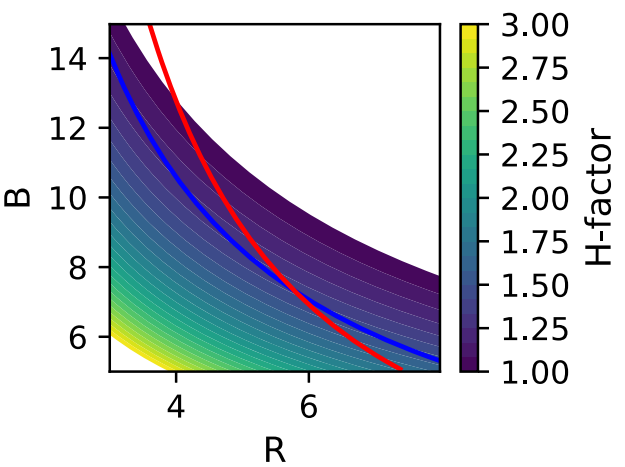

$\mathrm{H}$-factor has very high values in excess of 2 . It seems impossible to find, with our assumptions, a set of parameters in which all 3 quantities $H, \beta_{N}$ and $f_{G W}$ have conservative values and the operation point represents an attractive steady state tokamak in terms of $Q_{P B}$ and $Q_{C D}$ as well as the exhaust criterion (10).

In conclusion, we have shown that the possibility to build tokamaks at higher field than is presently possible calls for an optimization procedure that is not necessarily similar to that applied for present designs of reactor-grade devices. We have proposed a procedure to obtain design points which are steady state and fulfill an exhaust similarity criterion with ITER, meaning that the exhaust scheme could be validated there. An important finding is, however, that attractive points in operational space, especially if they should be steady state, still require quite optimistic assumptions about the plasma performance in terms of $H$ and/or $\beta_{N}$. This is also evident from previous studies of high field devices [2, 3, 5].

Finally, we remind the reader that a rigorous technology $R \& D$ programme will be needed to solve the presently unresolved issues, such as high mechanical forces or neutron shielding at reduced radial build, if the high field approach should be pursued in future.

Acknowledgements Open access funding provided by Max Planck Society. The author wants to acknowledge enlightening discussions with V. Chan, E. Fable, T. Pütterich and M. Siccinio as well as the programming support by A. Bock. The help of P. Bonoli with the comparison to ARC is also gratefully acknowledged. This work has been carried out within the framework of the EUROfusion
Consortium and has received funding from the Euratom research and training programme 2014-2018 under Grant Agreement Number 633053. The views and opinions expressed herein do not reflect those of the European Commission.

Open Access This article is distributed under the terms of the Creative Commons Attribution 4.0 International License (http://creative commons.org/licenses/by/4.0/), which permits unrestricted use, distribution, and reproduction in any medium, provided you give appropriate credit to the original author(s) and the source, provide a link to the Creative Commons license, and indicate if changes were made.

\section{References}

1. H. Zohm, Fusion Sci. Technol. 58, 613 (2010)

2. A. Costley et al., Nucl. Fusion 56, 066003 (2017)

3. W. Biel et al., Nucl. Fusion 57, 038001 (2017)

4. G.F. Federici et al., Fusion Eng. Des. 89, 882 (2014)

5. B. Sorbom et al., Fusion Eng. Des. 100, 378 (2015)

6. H. Zohm et al., Nucl. Fusion 57, 086002 (2017)

7. H.-S. Bosch, M. Hale, Nucl. Fusion 32, 611 (1992)

8. E. Fable et al., Nucl. Fusion 57, 022015 (2017)

9. N. Uckan et al ITER physics design guidelines: 1989, ITER documentation series 10 (Vienna: IAEA), based on B.A. Trubnikov, Rev. Plasma Physics 7 (1979), ed. M. Leontovich (New York: Plenum Press) p. 345

10. E. Dyole et al., Nucl. Fusion 47, S18 (2010)

11. A. Bock et al., Nucl. Fusion 57, 126041 (2017)

12. F. Turco et al., Phys. Plasmas 22, 056113 (2015)

13. A. Garofalo et al., Nucl. Fusion 55, 123025 (2015)

14. Y.X. Wan, Nucl. Fusion 57, 102009 (2017)

15. M. Siccinio et al., Nucl. Fusion 58, 016032 (2018)

16. M.L. Reinke, Nucl. Fusion 57, 034004 (2017) 